\title{
Life expectancy of type 1 diabetic patients during 1997-2010: a national Australian registry-based cohort study
}

\author{
Lili Huo $^{1,2}$ • Jessica L. Harding ${ }^{1,3}$ - Anna Peeters ${ }^{1,3}$ • Jonathan E. Shaw ${ }^{1,3}$. \\ Dianna J. Magliano ${ }^{1,3}$
}

Received: 10 September 2015 / Accepted: 14 December 2015 /Published online: 21 January 2016

(C) Springer-Verlag Berlin Heidelberg 2016

\begin{abstract}
Aims/hypothesis There is limited information about the impact of type 1 diabetes on life expectancy in a contemporary population. We examined the life expectancy of type 1 diabetic patients and explored the contribution of mortality at different ages and of different causes of death to years of life lost (YLL) compared with the general population.

Methods We derived mortality rates of Australians with type 1 diabetes listed on the National Diabetes Services Scheme (NDSS) between 1997 and $2010(n=85,547)$ by linking the NDSS to the National Death Index. The Chiang method was used to estimate life expectancy and Arriaga's method was used to estimate the contributions of age-specific and causespecific mortality to the YLL.

Results A total of 5,981 deaths were identified during the 902,136 person-years of follow up. Type 1 diabetic patients had an estimated life expectancy at birth of 68.6 years $(95 \%$ CI 68.1, 69.1), which was 12.2 years $(95 \%$ CI $11.8,12.7)$ less
\end{abstract}

Jonathan E. Shaw and Dianna J. Magliano are joint senior authors on this paper.

Electronic supplementary material The online version of this article (doi:10.1007/s00125-015-3857-4) contains peer-reviewed but unedited supplementary material, which is available to authorised users.

Lili Huo

lili.huo@bakeridi.edu.au

1 Department of Clinical Diabetes and Epidemiology, Baker IDI Heart and Diabetes Institute, Level 4, 99 Commercial Road,

Melbourne, VIC 3004, Australia

2 Department of Endocrinology, Beijing Jishuitan Hospital, Beijing, China

3 Department of Epidemiology and Preventive Medicine, Monash University, Melbourne, VIC, Australia than that in the general population. The improvement in life expectancy at birth in 2004-2010 compared with 1997-2003 was similar for both type 1 diabetic patients (men, 1.9 years [95\% CI 0.4 3.3]; women, 1.5 years [95\% CI 0.0, 3.2]) and the general population (men, 2.2 years; women, 1.4 years). Deaths at age $<60$ years accounted for $60 \%$ of the YLL from type 1 diabetes for men and $45 \%$ for women. The major contribution to YLL was mortality from endocrine and metabolic disease at age 10-39 years (men, 39-59\%; women, 35-50\%) and from circulatory disease at age $\geq 40$ years (men, $43-75 \%$; women, 34-75\%).

Conclusions/interpretation Data from 1997 to 2010 showed that Australian type 1 diabetic patients had an estimated loss in life expectancy at birth of 12.2 years compared with the general population.

Keywords Life expectancy - Type 1 diabetes · Years of life lost

$\begin{array}{ll}\text { Abbreviations } \\ \text { AIHW } & \text { Australian Institute of Health and Welfare } \\ \text { IQR } & \text { Interquartile range } \\ \text { NDSS } & \text { National Diabetes Services Scheme } \\ \text { NDI } & \text { National Death Index } \\ \text { PY } & \text { Person-year } \\ \text { YLL } & \text { Years of life lost }\end{array}$

\section{Introduction}

Type 1 diabetes is associated with a substantially increased risk of premature death. The survival of type 1 diabetic patients has recently improved with advances in treatment [1-4]. However, mortality is still two to five times higher for this 
population compared with the general population [3-7]. Suboptimal glycaemic control and the presence of acute and chronic complications are key contributors to reduced life expectancy for type 1 diabetic patients $[2,6]$.

In 1975, Goodkin reported a loss of 27 years for diabetic patients compared with individuals without diabetes [8]. In the following two decades, several studies reported that years of life lost (YLL) due to type 1 diabetes had fallen to 15-20 years $[5,9,10]$. More recently, a US study with 30 years of follow up showed that type 1 diabetic patients diagnosed between 1965 and 1980 had a life expectancy of 68.8 years at birth, approximately 15 years more than those diagnosed between 1950 and 1964 [11]. The gap between life expectancy for type 1 diabetic patients (diagnosed between 1965 and 1980) and the general US population was just 3.8 years, but this study only focused on childhood onset type 1 diabetes (age $<17$ years). In contrast, Livingstone et al investigated the life expectancy of nearly 25,000 type 1 diabetic patients in Scotland between 2008 and 2010 [12]. They found that 20-year-old women and men with type 1 diabetes could expect to live for 12.9 and 11.1 years, respectively, less than aged-matched adults without diabetes; however, this study only included patients $\geq 20$ years old and was based on only 3 years of data.

The number of studies estimating life expectancy for type 1 diabetes under contemporary care is very limited. Furthermore, little is known about which diseases contribute most to YLL, and whether loss of life expectancy is driven by a small number of very premature deaths in young people, a larger number of deaths occurring in middle-aged and older adults or both. Such information is important for understanding how and where to direct interventions to improve life expectancy. We explored these issues using a large national Australian registry of type 1 diabetic patients.

\section{Methods}

Data sources The National Diabetes Services Scheme (NDSS) was established by the Australian government in 1987 to deliver diabetes-related products at subsidised prices and provide information to diabetic patients. Patient registration is free and completed by a medical practitioner or credentialed diabetes nurse educator. The NDSS includes almost $100 \%$ of type 1 diabetic patients [13]. We studied type 1 diabetic patients who were listed on the NDSS between 1997 and 2010, including all prevalent cases on 1 January 1997 and incident cases between 1 January 1997 and 31 December 2010 (electronic supplementary material [ESM] Table 1). The year 1997 was chosen as the start date because it followed the unification of state-based registries and an improvement in data quality.

Diabetes type is classified by the health practitioner at registration. However, for the current study, type 1 diabetes status was assigned to registrants recorded as type 1 on the NDSS registry who were diagnosed at age $<45$ years and taking insulin. The registration date was used as a proxy for the diagnosis date because the diagnosis date was missing for a large proportion of registrants $(56.2 \%)$. We chose 45 years as the cut-off to minimise the number of missed type 1 diabetic patients without misclassifying significant numbers of patients with type 2 diabetes as having type 1 [14]. Additionally, those registrants recorded as having type 2 diabetes who were diagnosed before age 30 years and were taking insulin within 1 year of the diagnosis date were reclassified as having type 1 diabetes [12].

The NDSS was linked to the National Death Index (NDI) using data up to and including 31 December 2010. Linkage was performed by the Australian Institute of Health and Welfare (AIHW) as previously reported $[15,16]$. Data for the general Australian population between 1997 and 2010 were provided by the AIHW.

The underlying cause of death was classified according to the WHO ICD-10 (www.who.int/classifications/icd/en/) into eight categories: circulatory disease, I00-I99; neoplasm, C00-D48; genitourinary disease, N00-N99; infectious disease, A00-B99; respiratory disease, J00-J99; injury, V01-Y98; endocrine and metabolic disease, E00-E90; and others. Deaths for which the underlying cause corresponded to 'uncomplicated diabetes' (E10.9, E11.9, E12.9, E13.9, E14. 9) or 'diabetes with circulatory complications' (E10.5, E11.5, E12.5, E13.5, E14.5) and where a ICD-10 code for circulatory disease also appeared in the first line of part I of the death certificate were included in the circulatory disease group. These are thought to reflect deaths due to circulatory disease because people are unlikely to die of 'uncomplicated diabetes' or 'diabetes with circulatory complications'; in such cases, circulatory disease is probably a consequence of diabetes [4].

Statistical analysis Individuals were followed up from 1 January 1997, or the registration date if thereafter, to 31 December 2010 or the date of death, whichever occurred first. Age-specific all-cause mortality rates (by 5-year intervals) were calculated stratified by sex and time period (19972003, 2004-2010). We also calculated the age-specific allcause mortality rates by age at diagnosis $(0-14,15-29$ and $\geq 30$ years) for those for whom the date of diagnosis was available $(43.8 \% ; n=37,472)$. Mortality data for the general population were available by 5 -year age intervals stratified by sex and different time periods (1997-2003; 2004-2010).

Life expectancy at an exact age is defined as the average remaining years of life of a group after that age, according to the age-specific all-cause mortality rates for the study period. Abridged period life tables were constructed using the Chiang method [17] by 5 -year age intervals up to age 84 years and an open-ended interval thereafter (conditional on surviving to the diagnosis date for the type 1 diabetic population). Age- 
specific mortality rates for both populations were applied to the life tables. Life tables were constructed for the total population, for men and women, and for different year intervals to estimate the remaining life expectancy, with the assumption that variations in diabetes duration has a small effect on the life expectancy at an attained age. For example, the life expectancy of type 1 diabetic patients at age 20 years is the average remaining years that will be lived after age 20 years by the population on the NDSS with type 1 diabetes. To assess the potential effect of the diabetes duration, we also constructed life tables by age at diagnosis. For age intervals for which there were no data, the mortality rate was assumed to be that of the general population. CIs were calculated using Monte Carlo simulations [18]. Arriaga's method was used to estimate the contributions of age-specific or cause-specific mortality stratified by age to the YLL for type 1 diabetic patients compared with the general population $[19,20]$. The contribution of each cause can be negative because of higher cause-specific mortality in the general population.

Sensitivity analyses were performed to examine the effects of choosing different cut-offs of age at diagnosis $(<30$ and $<40$ years) and of excluding those without a diagnosis date. We also estimated the life expectancy between 2008 and 2010.

Data analysis was carried out using Stata 12.1 (StataCorp, College Station, TX, USA) and Excel 2007 (Microsoft, Redmond, WA, USA). This study was approved by the Alfred Health Human Ethics Committee and the AIHW Ethics Committee.

\section{Results}

Study characteristics The study included 85,547 type 1 diabetic patients who were listed on the NDSS between 1997 and 2010. The median age at registration was 26.9 years (interquartile range [IQR] 14.6-36.2) and median follow-up was 13.8 years (IQR 7.2-14.0). For those with a diagnosis date, the median age at diagnosis was 20.4 years (IQR 11.2-30.8). A total of 5,981 deaths (62.5\% among men) were identified during the 902,136 person-years (PYs) of follow-up. The median age at death was 50.6 years (IQR 42.8-57.3) and the median diabetes duration at death was 14.5 years (IQR 10.0-19.4). The crude mortality rates (per 1,000 PYs) were $8.1(95 \%$ CI $7.9,8.4)$ and $5.1(95 \%$ CI $4.9,5.3)$ for men and women, respectively (Table 1).

Estimated life expectancy Survival was worse in the type 1 diabetic population compared with the general population (Fig. 1). In the general population, $79.5 \%$ of men and $87.7 \%$ of women survived to age 70 years, while the corresponding percentages were $53.8 \%$ for men and $64.1 \%$ for women in the type 1 diabetic population. Type 1 diabetic patients had an estimated life expectancy at birth of 68.6 years $(95 \%$ CI
$68.1,69.1)$, which was 12.2 years $(95 \%$ CI $11.8,12.7)$ less than that of the general population. For type 1 diabetic men, the estimated life expectancy at birth and loss in life expectancy compared with the general population were 66.7 years (95\% CI 66.1, 67.5) and 11.6 years $(95 \%$ CI 10.9, 12.2), respectively (Table 2). For type 1 diabetic women, these estimates were 70.9 years $(95 \%$ CI $70.3,71.7)$ and 12.5 years (95\% CI 11.7, 13.1), respectively. The reduction in life expectancy gradually declined with increasing age for both sexes.

Over the 14-year follow-up, life expectancy improved to a similar extent for both type 1 diabetic patients and the general population (ESM Tables 2 and 3). From the first (1997-2003) to the second (2004-2010) time interval, the estimated life expectancy at birth increased by 1.9 years $(95 \%$ CI $0.4,3.3)$ for type 1 diabetic men and by 1.5 years $(95 \%$ CI $0.0,3.2)$ for type 1 diabetic women (ESM Table 2$)$. The corresponding improvements in the general population were 2.2 years for men and 1.4 years for women (ESM Table 3).

There was a slight improvement in estimated life expectancy with increasing age at type 1 diabetes diagnosis. For example, at the age of 35 years, patients diagnosed before age 15 years lived 1.6 fewer years compared with those diagnosed between the ages of 15 and 29 years (32.6 years [95\% CI 30.2, $35.4]$ vs 34.2 years [95\% CI 32.9, 35.9]) and 2.5 fewer years than those diagnosed at age $>30$ years $(32.6$ years $[95 \% \mathrm{CI}$ $30.2,35.4$ ] vs 35.1 years [95\% CI 34.5, 35.8]). However, these differences were reduced at an attained age $>50$ years (ESM Table 4).

Sensitivity analyses In the sensitivity analyses, when the cutoff for age at diagnosis (or registration, when this data was missing) was chosen as $<30$ and $<40$ years, the estimated life expectancy at birth for the type 1 diabetic population was 67.4 years $(95 \%$ CI $66.2,68.7)$ and 68.5 years $(95 \% \mathrm{CI}$ $67.9,69.2)$, respectively, which was 1.2 years $(95 \% \mathrm{CI}-0.1$, $2.7)$ and 0.1 years $(95 \% \mathrm{CI}-0.7,0.9)$ fewer than the result obtained with a cut-off of $<45$ years (ESM Tables 5 and 6). Analysis of data restricted to patients with a known diagnosis date found the estimated life expectancy at birth to be 67.9 years (95\% CI 67.3, 68.6; ESM Tables 7 and 8). Additionally, when the analysis was restricted to data from 2008-2010, type 1 diabetic patients had an estimated life expectancy at birth of 70.0 years $(95 \%$ CI $69.0,71.1)$ and the YLL $(12.0$ years $[95 \%$ CI 11.0, 13.1]) was similar to that of the period 1997-2010 (ESM Table 9).

Examination of YLL by age and cause of death The decomposition of YLL by age group is shown in Fig. 2. For men, deaths under the age of 40 and 60 years were responsible for $21.1 \%$ and $60.2 \%$, respectively, of the total YLL. The percentage contribution of death in each age group to the total YLL increased with increasing age until age 45-49 years and declined thereafter. For women, deaths under the ages of 40 and 
Table 1 Characteristic of the participants with type 1 diabetes during 1997-2010

\begin{tabular}{|c|c|c|c|c|c|c|}
\hline Sex & $n$ & All deaths $(n)$ & $\begin{array}{l}\text { Age at registration } \\
(\text { years })^{\mathrm{a}}\end{array}$ & $\begin{array}{l}\text { Age at diagnosis } \\
{\text { (years })^{\text {a, b }}}\end{array}$ & $\begin{array}{l}\text { Diabetes duration } \\
\text { at last follow- } \\
\text { up (years) }{ }^{\mathrm{a}, \mathrm{b}}\end{array}$ & $\begin{array}{l}\text { Mortality rate } \\
(95 \% \text { CI })\end{array}$ \\
\hline Men & 44,892 & 3,740 & $27.5(15.0-37.1)$ & $20.9(11.7-31.7)$ & $8.2(4.0-12.5)$ & $8.1(7.9,8.4)$ \\
\hline Women & 40,655 & 2,241 & $26.6(14.1-35.3)$ & $19.9(10.6-29.9)$ & $9.0(4.6-12.9)$ & $5.1(4.9,5.3)$ \\
\hline Total & 85,547 & 5,981 & $26.9(14.6-36.2)$ & $20.4(11.2-30.8)$ & $8.6(4.3-12.7)$ & $6.6(6.5,6.8)$ \\
\hline
\end{tabular}

${ }^{a}$ Data are medians (IQR)

${ }^{\mathrm{b}}$ Including only those with a known diagnosis date

${ }^{\mathrm{c}}$ Expressed per 1,000 PYs

60 years contributed $15.1 \%$ and $45.4 \%$, respectively, to the total YLL. There was a similar rise with increasing age, but the contribution plateaued, rather than fell, after the age of 50 years.

Overall, circulatory disease was the leading cause of death in type 1 diabetic patients (accounting for $37.5 \%$ of all deaths and for almost $50 \%$ of all YLL), followed by endocrine and metabolic disease (accounting for $20.7 \%$ of all deaths and almost $30 \%$ of all YLL) (Fig. 3). Above the age of 40 years,
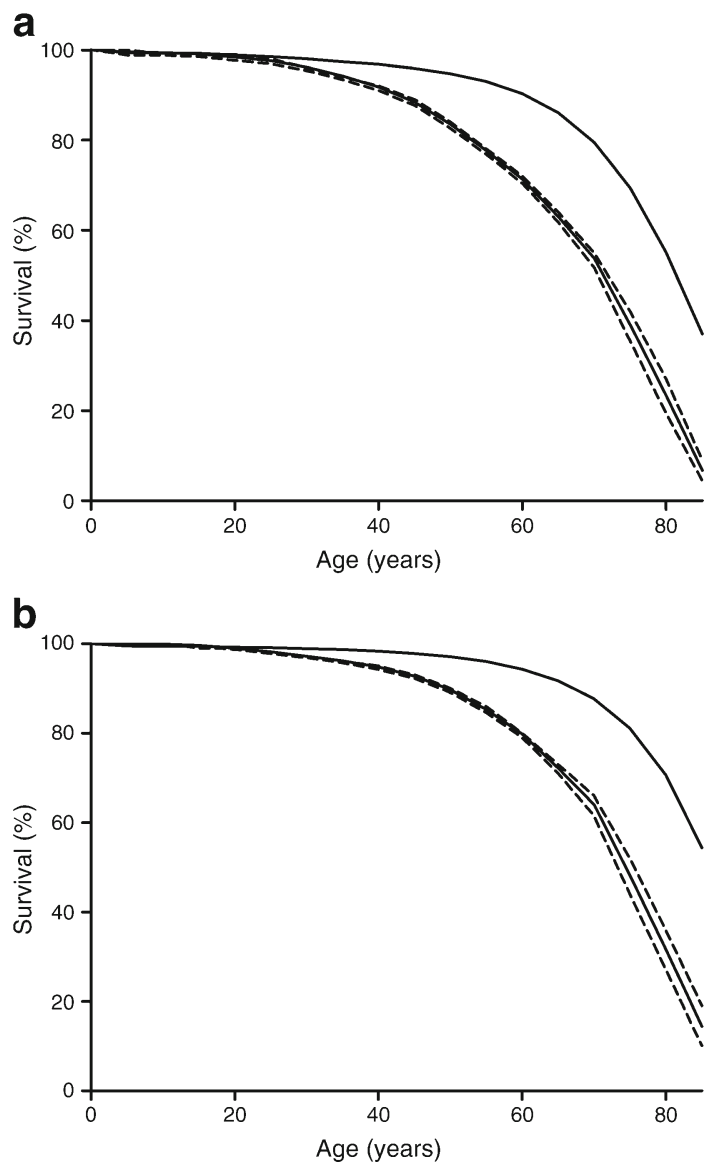

Fig. 1 Percentage survival by age for (a) men and (b) women with type 1 diabetes compared with the general population during 1997-2010. Solid line, general population; solid line with a dotted line on each side, type 1 diabetic patients, the dotted lines represent the $95 \%$ CIs circulatory disease accounted for $40-60 \%$ of deaths, followed by neoplasm (which accounted for $10-20 \%$ of deaths). Endocrine and metabolic disease was the most common cause of deaths occurring between the ages of 10 and 40 years (at $30-40 \%$ ); of these, diabetic coma, hypoglycaemia and diabetic ketoacidosis accounted for $35-60 \%$. Figure 4 shows the contribution of each cause of death to the YLL by age. At ages 10-39 years, mortality from endocrine and metabolic disease made the greatest contribution to the YLL. This peaked at age 10-19 years, with $59.4 \%$ for men and $50.2 \%$ for women. The contribution of mortality from endocrine and metabolic disease decreased with increasing age, but was still important in the YLL of elderly people. In contrast, the contribution of mortality caused by circulatory disease to the YLL increased with increasing age (43-75\% for men and 34-75\% for women aged $\geq 40$ years) and peaked at age $70-79$ years $(75.0 \%$ for men and $74.6 \%$ for women). Circulatory disease contributed 5.7 YLL for men and 6.2 YLL for women, and endocrine and metabolic disease contributed 3.3 YLL for both men and women. Another important contributor was death from neoplasm between the ages of 10 and 50 years, which explained $4-20 \%$ and $7-14 \%$ of the YLL for men and women, respectively. Death from neoplasm at age $>50$ years had a much lower contribution to the total YLL.

\section{Discussion}

In an Australian national, population-based cohort followed from 1997 to 2010, we estimated the overall life expectancy of type 1 diabetic patients to be 12.2 years less than that of the general population. The life expectancy was reduced for both sexes and across all age groups. For type 1 diabetic patients, the estimated life expectancy at birth was 66.7 and 70.9 years for men and women, respectively, representing 11.6 and 12.5 years less than that of the general population, respectively. Decomposition of the YLL for type 1 diabetic patients showed that mortality from endocrine and metabolic disease made the largest contribution to YLL in the 10-39 year age interval and decreased with increasing age, while the 
Table 2 Abridged period life table for men and women with type 1 diabetes vs general population in Australia during 1997-2010

\begin{tabular}{|c|c|c|c|c|c|}
\hline \multirow[t]{2}{*}{$\begin{array}{l}\text { Age interval } \\
\text { (years) }\end{array}$} & \multicolumn{2}{|c|}{ Type 1 diabetes } & \multicolumn{3}{|c|}{$\begin{array}{l}\text { Estimated remaining life expectancy (years) } \\
(95 \% \mathrm{CI})^{\mathrm{b}}\end{array}$} \\
\hline & $\begin{array}{l}\text { Observed } \\
\text { deaths }\end{array}$ & $\begin{array}{l}\text { Mortality rate } \\
(95 \% \mathrm{CI})^{\mathrm{a}}\end{array}$ & $\begin{array}{l}\text { Type } 1 \text { diabetic } \\
\text { patients }\end{array}$ & $\begin{array}{l}\text { General } \\
\text { population }\end{array}$ & $\begin{array}{l}\text { Difference in life } \\
\text { expectancy }\end{array}$ \\
\hline \multicolumn{6}{|l|}{ Men } \\
\hline $0-4$ & 3 & $1.0(0.3,3.2)$ & $66.7(66.1,67.5)$ & $78.3(78.3,78.3)$ & $11.6(10.9,12.2)$ \\
\hline $5-9$ & 2 & $0.2(0.0,0.7)$ & $62.1(61.6,62.6)$ & $73.8(73.8,73.8)$ & $11.7(11.2,12.2)$ \\
\hline $10-14$ & 12 & $0.5(0.3,0.9)$ & $57.1(56.7,57.7)$ & $68.9(68.8,68.9)$ & $11.7(11.2,12.2)$ \\
\hline $15-19$ & 46 & $1.5(1.1,1.9)$ & $52.3(51.8,52.8)$ & $63.9(63.9,63.9)$ & $11.6(11.1,12.1)$ \\
\hline $20-24$ & 57 & $1.6(1.3,2.1)$ & $47.6(47.1,48.2)$ & $59.1(59.1,59.1)$ & $11.5(10.9,11.9)$ \\
\hline $25-29$ & 117 & $2.9(2.4,3.5)$ & $43.0(42.5,43.6)$ & $54.4(54.3,54.4)$ & $11.3(10.8,11.8)$ \\
\hline $30-34$ & 199 & $4.3(3.7,4.9)$ & $38.6(38.1,39.2)$ & $49.6(49.6,49.6)$ & $11.0(10.4,11.5)$ \\
\hline $35-39$ & 270 & $5.2(4.6,5.8)$ & $34.4(33.9,35.0)$ & $44.9(44.9,44.9)$ & $10.5(9.9,11.0)$ \\
\hline $40-44$ & 414 & $7.4(6.7,8.1)$ & $30.2(29.7,30.8)$ & $40.2(40.2,40.2)$ & $10.0(9.4,10.4)$ \\
\hline $45-49$ & 663 & $11.5(10.6,12.4)$ & $26.3(25.7,26.8)$ & $35.5(35.5,35.5)$ & $9.2(8.7,9.8)$ \\
\hline $50-54$ & 741 & $14.5(13.5,15.6)$ & $22.7(22.1,23.3)$ & $30.9(30.9,30.9)$ & $8.2(7.7,8.8)$ \\
\hline $55-59$ & 571 & $17.2(15.8,18.6)$ & $19.2(18.6,19.8)$ & $26.4(26.4,26.5)$ & $7.3(6.6,7.8)$ \\
\hline $60-64$ & 355 & $24.6(22.2,27.4)$ & $15.7(15.1,16.3)$ & $22.1(22.1,22.2)$ & $6.5(5.8,7.0)$ \\
\hline $65-69$ & 100 & $31.5(25.9,38.3)$ & $12.4(11.8,13.2)$ & $18.1(18.1,18.1)$ & $5.7(4.9,6.3)$ \\
\hline $70-74$ & 67 & $63.8(50.2,81.1)$ & $9.1(8.5,9.9)$ & $14.4(14.4,14.4)$ & $5.3(4.5,5.9)$ \\
\hline $75-79$ & 56 & $100.2(77.1,130.2)$ & $6.6(6.0,7.4)$ & $11.1(11.1,11.2)$ & $4.5(3.8,5.1)$ \\
\hline $80-84$ & 49 & $221.2(167.1,292.6)$ & $4.4(3.8,5.1)$ & $8.4(8.4,8.4)$ & $4.0(3.3,4.6)$ \\
\hline$\geq 85$ & 18 & $252.2(158.9,400.3)$ & $4.0(3.5,4.4)$ & $6.3(6.2,6.3)$ & $2.3(1.9,2.7)$ \\
\hline \multicolumn{6}{|l|}{ Women } \\
\hline $0-4$ & 0 & 0.0 & $70.9(70.3,71.7)$ & $83.4(83.4,83.4)$ & $12.5(11.7,13.1)$ \\
\hline $5-9$ & 3 & $0.3(0.1,0.8)$ & $65.9(65.3,66.6)$ & $78.8(78.8,78.8)$ & $12.9(12.2,13.5)$ \\
\hline $10-14$ & 14 & $0.6(0.4,1.0)$ & $61.0(60.3,61.8)$ & $73.8(73.8,73.9)$ & $12.8(12.0,13.5)$ \\
\hline $15-19$ & 35 & $1.2(0.9,1.7)$ & $56.2(55.6,56.9)$ & $68.9(68.9,68.9)$ & $12.7(12.0,13.3)$ \\
\hline $20-24$ & 53 & $1.6(1.2,2.1)$ & $51.5(50.8,52.2)$ & $64.0(64.0,64.0)$ & $12.5(11.7,13.1)$ \\
\hline $25-29$ & 79 & $2.0(1.6,2.5)$ & $46.9(46.2,47.6)$ & $59.1(59.1,59.1)$ & $12.2(11.5,12.9)$ \\
\hline $30-34$ & 107 & $2.2(1.8,2.6)$ & $42.3(41.6,43.1)$ & $54.2(54.2,54.2)$ & $11.8(11.1,12.5)$ \\
\hline $35-39$ & 170 & $2.9(2.5,3.4)$ & $37.8(37.1,38.5)$ & $49.3(49.3,49.3)$ & $11.5(10.8,12.2)$ \\
\hline $40-44$ & 261 & $4.4(3.9,4.9)$ & $33.3(32.6,34.1)$ & $44.5(44.4,44.5)$ & $11.2(10.4,11.8)$ \\
\hline $45-49$ & 362 & $6.7(6.0,7.4)$ & $29.0(28.3,29.8)$ & $39.7(39.7,39.7)$ & $10.7(9.9,11.4)$ \\
\hline $50-54$ & 419 & $10.0(9.1,11.0)$ & $24.9(24.2,25.7)$ & $35.0(34.9,35.0)$ & $10.1(9.2,10.7)$ \\
\hline $55-59$ & 331 & $13.3(11.9,14.8)$ & $21.0(20.3,21.9)$ & $30.3(30.3,30.3)$ & $9.3(8.5,10.1)$ \\
\hline $60-64$ & 209 & $20.0(17.5,22.9)$ & $17.3(16.6,18.2)$ & $25.8(25.8,25.8)$ & $8.5(7.6,9.3)$ \\
\hline $65-69$ & 54 & $23.7(18.1,30.9)$ & $13.8(13.0,14.8)$ & $21.5(21.5,21.5)$ & $7.6(6.6,8.4)$ \\
\hline $70-74$ & 49 & $56.9(43.0,75.3)$ & $10.3(9.4,11.3)$ & $17.4(17.3,17.4)$ & $7.1(6.1,8.0)$ \\
\hline $75-79$ & 45 & $82.3(61.5,110.2)$ & $7.9(7.0,8.8)$ & $13.6(13.6,13.6)$ & $5.7(4.8,6.6)$ \\
\hline $80-84$ & 31 & $150.0(105.5,213.4)$ & $5.6(4.8,6.6)$ & $10.2(10.2,10.2)$ & $4.6(3.6,5.4)$ \\
\hline$\geq 85$ & 19 & $228.2(145.6,357.7)$ & $4.4(3.8,5.0)$ & $7.5(7.5,7.5)$ & $3.1(2.5,3.7)$ \\
\hline
\end{tabular}

${ }^{\mathrm{a}}$ Expressed per 1,000 PYs

${ }^{\mathrm{b}}$ Represents the average remaining life expectancy at each attained age contribution of mortality from circulatory disease increased with increasing age.

Few studies have focused on life expectancy in a representative type 1 diabetic population. This study used data from a nationwide register of type 1 diabetes with an almost 100\% capture rate. We observed marked reductions in life expectancy across all age intervals, even in the very old, as compared with the general population. Our results are comparable with those of a recent study from Scotland [12] which estimated a reduction in life expectancy of 11.1 years for men with type 1 
Fig. 2 Age-specific contributions to the YLL in type 1 diabetic patients during 19972010. Black bars, men; white bars, women

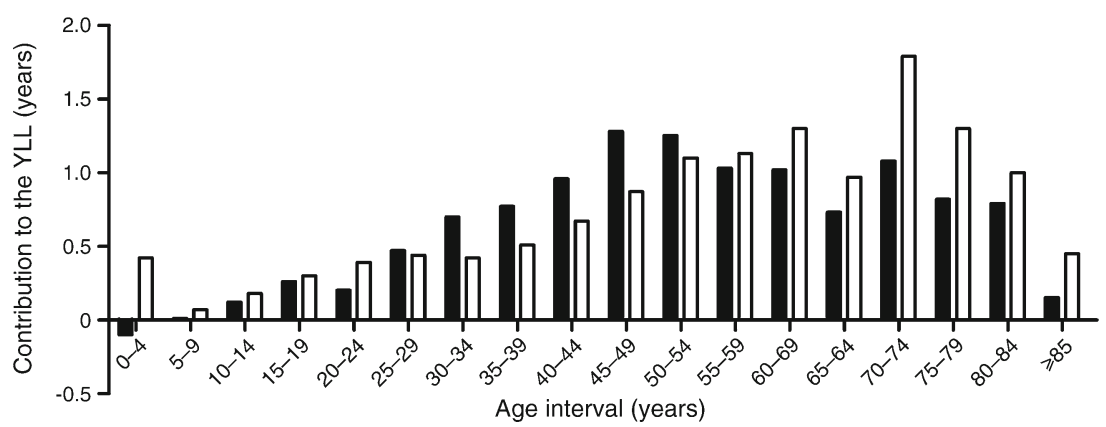

diabetes and 12.9 years for women with type 1 diabetes at an attained age of 20 years. Despite the similar YLL of participants in these two studies, the higher mortality of type 1 diabetic patients aged 75 or older explains a larger proportion of the YLL in our study (15.3\% for men and $22.1 \%$ for women) than in the Scottish study (4.6\% for men and $13.4 \%$ for women). Australian type 1 diabetic patients have higher mortality rates than their Scottish counterparts at old and very old ages, although they do considerably better at younger ages.

Several studies have shown that calendar time periods, which are used as surrogates for different treatment eras, were associated with differential survival times $[1,2,4,11]$. A 30 -year follow up study by Miller et al showed a dramatic increase in life expectancy in type 1 diabetic patients over time [11]. The life expectancy at birth for those diagnosed in 19651980 was 68.8 years (which is comparable to 68.6 years for both sexes combined found in our study), but with a substantial increase of 15 years in life expectancy compared with individuals diagnosed in 1950-1964. We divided the 14-year follow-up into two periods (1997-2003 and 20042010), and found an improvement of nearly 2 years in the life expectancy of type 1 diabetic patients. However, a similar increment of life expectancy was found in the general population, indicating that there was no improvement in loss of life due to type 1 diabetes. In a Romanian type 1 diabetes cohort [1], the mean age at death increased by 7 years for patients diagnosed at age $<18$ years and 12 years for patients diagnosed at age $\geq 18$ years when comparing those diagnosed in 1966-1985 with those diagnosed in 1946-1965 [11]. The improvement continued into the next period (1986-2005) for patients diagnosed at age $<18$ years, but was much reduced in patients diagnosed at age $\geq 18$ years. Our study population has a relatively high age of diagnosis (around 20 years), which may have contributed to the smaller improvement in life expectancy over time compared with the study by Miller et al, which focused only on childhood onset diabetes [11]. In addition, Miller and colleague's study began much earlier when mortality from diabetes and cardiovascular disease was much higher [3]; thus, the participants might have benefited more from advances in treatment.

Age at diabetes onset plays a critical role in determining the risk of developing diabetic complications and may thus affect the mortality rate and life expectancy. Our study shows a slight improvement in the estimated life expectancy with increasing age at diagnosis. This is similar to the findings of Brown et al [5]. The interrelationship among attained age, age at onset and duration of diabetes makes it difficult to separate the impact of age at onset on life expectancy from the impact of diabetes duration.

In this study, we partitioned the YLL from type 1 diabetes into causes of death at different ages. Data on the underlying cause of death were taken from death certificates and the
Fig. 3 Age distribution of causes of death in type 1 diabetic patients between 1997 and 2010. End + met, endocrine and metabolic

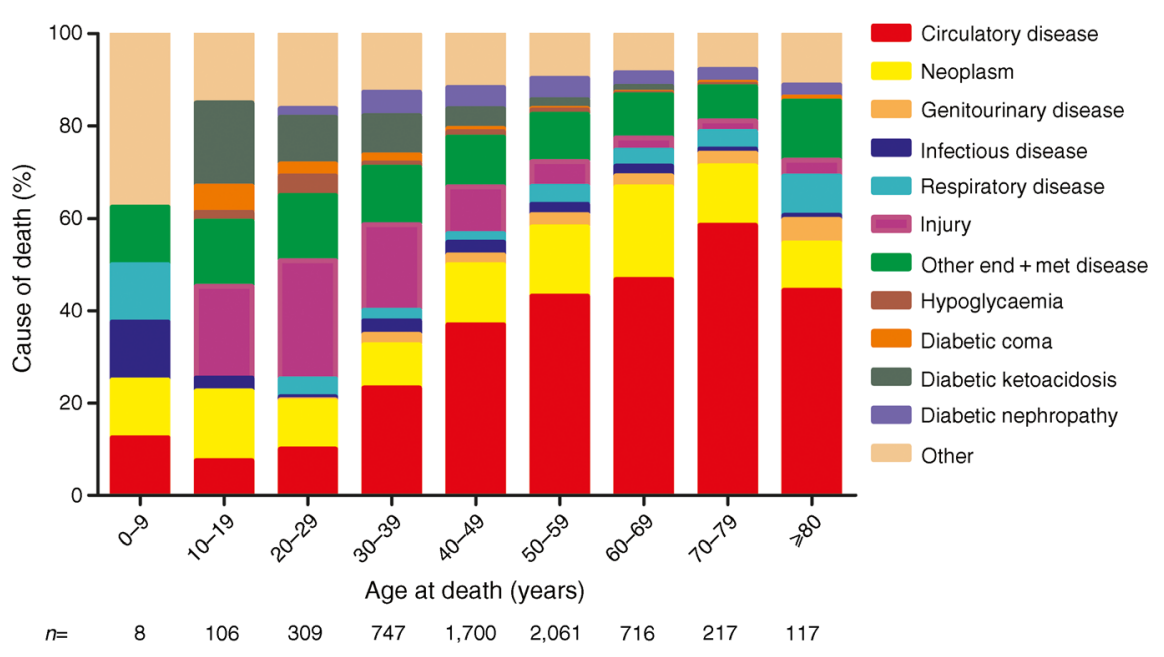


Fig. 4 Cause-specific contributions to the YLL stratified by age for (a) men and (b) women with type 1 diabetes between 1997 and 2010. End + met, endocrine and metabolic
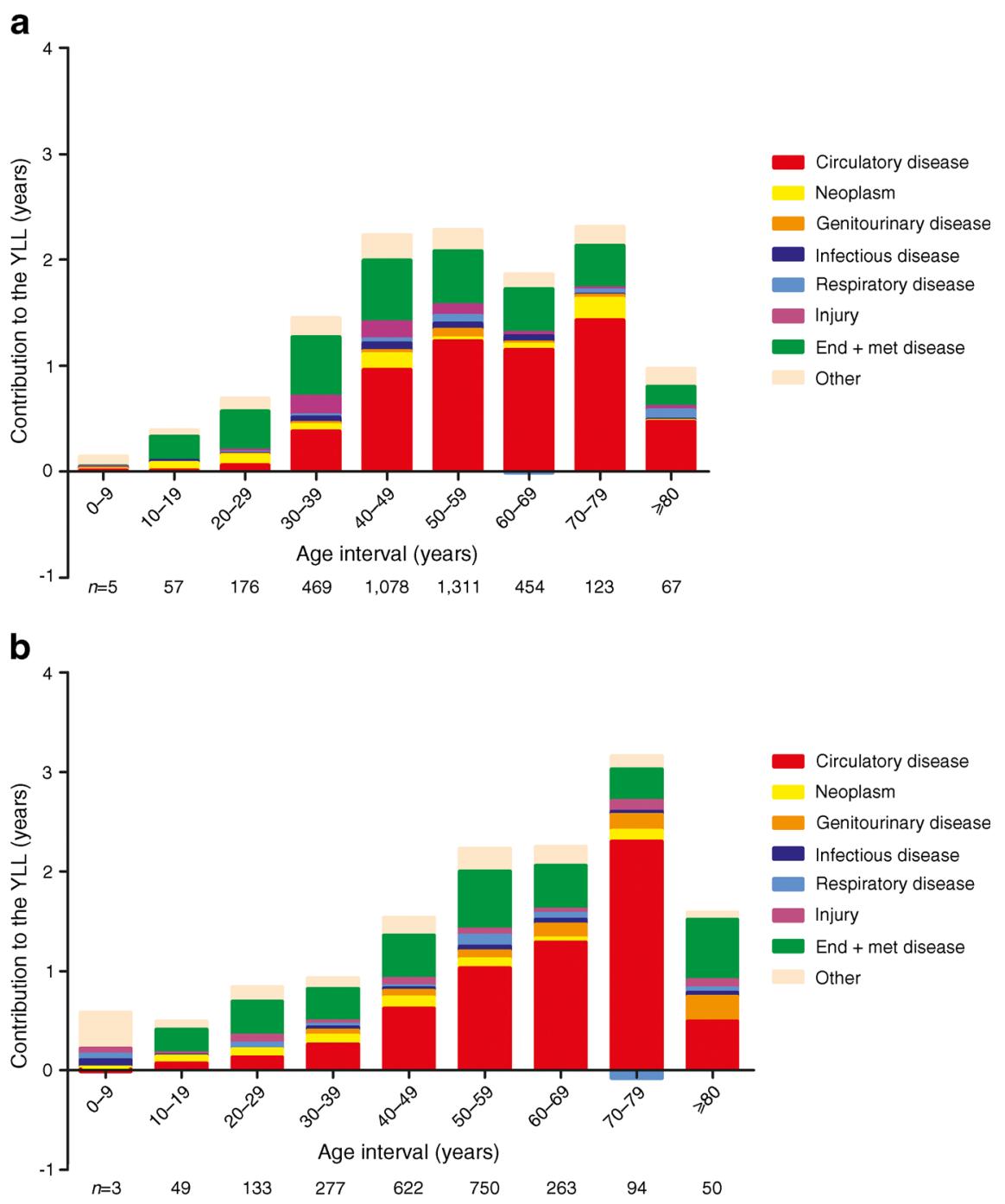

ICD-10 codes, which may have some errors. We previously showed that the use of traditional coding methods for the underlying cause of death led to $39 \%$ of cardiovascular deaths being misclassified in type 1 diabetes [4]. Therefore, in this study we recoded relevant 'uncomplicated diabetes' and 'diabetes with circulatory complications' deaths to circulatory disease if a circulatory disease ICD-10 code also appeared in the first line of part I of the death certificate. We found that circulatory disease and endocrine and metabolic disease were the major causes of death in type 1 diabetic patients. Several studies have demonstrated a decline in mortality from circulatory disease in recent decades in both diabetic and general populations $[3,4,21]$, although it is still a leading cause of death in type 1 diabetes, especially in the elderly $[2,12]$. The contribution of mortality from circulatory disease to the YLL also increased with increasing age, peaking at age 70-79 years for both sexes. Overall, excess mortality from circulatory disease was responsible for almost half of the total YLL (5.7 years for men; 6.2 years for women). These findings support a more aggressive management of circulatory disease in type 1 diabetic patients to prevent premature mortality. Mortality from endocrine and metabolic disease is a very important driver of the YLL, especially in early age intervals. We were unable to break down endocrine and metabolic disease into specific cause of death categories in the general population because the corresponding mortality data were unavailable. However, in the type 1 diabetic population, diabetic coma, hypoglycaemia and diabetic ketoacidosis comprised approximately $30 \%$ and diabetic nephropathy comprised $20 \%$ of all endocrine and metabolic deaths. Therefore, we believe that the mortality burden from endocrine and metabolic disease in type 1 diabetes probably reflects the large impact of acute complications and renal complications on YLL. Neoplasm was an important contributor to YLL between 10 and 50 years of age. This is consistent with previous findings by our group and others that type 1 diabetes is associated with a greater incidence of and mortality from overall cancer and some site-specific cancers [16, 22]. Individuals with type 1 diabetes may therefore benefit from more intensive cancer screening. 
As this is a contemporary nationwide registry-based cohort study of type 1 diabetes, the results are likely to be applicable to other similar Western countries. The key strengths of this study are the long follow-up period, the large national cohort, linkage to a national mortality registry and the accurate identification of cause of mortality. However, care is necessary when making inferences from life expectancy estimates. Period life table estimates are summary descriptive measures created from cross-sectional data for open populations with the main assumption of a steady-state. This means that the life expectancy for type 1 diabetes is estimated from data on type 1 diabetic patients with varying periods of disease exposure. In practice, estimates from this study cannot be used to 'predict' life expectancy; instead, they describe the mortality and health experiences of individuals with disease (in this case, type 1 diabetes) and in the general population. It should also be noted that a key assumption of this life table is that diabetes duration has a small effect. This is supported by data showing only small differences in life expectancy among groups with very different ages of diabetes diagnosis (ESM Table 4). Several limitations should also be considered. First, data for the general population pertain to the entire Australian population, including those with diabetes; thus, our analyses will underestimate the true YLL for type 1 diabetic patients. However, the effect will be small because of the low prevalence of type 1 diabetes $(0.4 \%$ in the Australian population) [23]. Other limitations arise from the potential misclassification of diabetes type. However, the age distribution and proportion of insulin usage is similar in the remaining type 2 diabetic patients in our dataset and in other populations [23, 24]; therefore, we do not think that any misclassification will significantly affect our results. The analysis stratified by age of diagnosis was restricted to the $44 \%$ of the cohort for whom a diagnosis date was available; however, this group may not fully represent the national type 1 diabetic cohort. Those registered in more recent periods (i.e. after 1999) were more likely to have complete information, including the diagnosis date. For those without a diagnosis date, we used the registration date as a proxy. This may have introduced bias due to missing some type 1 diabetic patients who were registered at age $\geq 45$ years. However, a sensitivity analysis showed that life expectancy was similar between those with a diagnosis date and the whole type 1 diabetic population. Cause of death is determined from the death certificate, and there is a long history of limited accuracy of death certificates [25-27]. This may be a problem for all the studies that rely on death certificate data. An Australian longitudinal cohort study found that almost $60 \%$ of the deaths with an underlying ICD code listed as 'diabetes' primarily resulted from cardiovascular disease [28]. Although we recoded 'uncomplicated diabetes' and 'diabetes with circulatory complications' deaths to circulatory disease in type 1 diabetes, we could not avoid the misclassification of some causes of death, especially at older ages. At these ages, comorbidities are very common and misclassification is more likely to occur; for example, kidney disease is underreported on death certificates. Misclassification may cause under- or overestimation of the contribution of a specific cause of death to the YLL but will not affect the overall findings on YLL from type 1 diabetes. Finally, the discrete method of Arriaga may underestimate the contribution of causes of death that are more common at older ages [20,29] because an initial decomposition by age is required before decomposition by cause. Furthermore, decomposition by cause of death assumes that each death has a single underlying cause, when, in reality, some deaths are due to several combined causes.

In summary, we have shown that Australians with type 1 diabetes had an estimated life loss of 11.6 years for men and 12.5 years for women compared with the general population. Early onset of diabetes tended to be a predictor of premature mortality. Deaths before the age of 60 years and mortality from circulatory disease and endocrine and metabolic disease contributed most to YLL in type 1 diabetes. For improvements in life expectancy, greater attention must therefore be paid to both the acute metabolic and chronic cardiovascular complications of type 1 diabetes. A failure to address either one will continue to leave type 1 diabetic patients at risk of premature mortality.

Acknowledgements We thank the NDSS, an initiative of the Australian government that has been administered by Diabetes Australia since 1987, for the source data, and thank the AIHW for linking the NDSS to the NDI to obtain mortality data. The interpretation and conclusions contained in this study are those of the authors alone.

Funding This study was funded by the National Health and Medical Research Council (NHMRC; APP1002663) and the Australian Government Department of Health and Ageing. This study was also partly supported by the Victorian Operational Infrastructure Program scheme. LH is supported by China Scholarship Council; JLH is supported by a Monash University Australian Postgraduate Award and a Baker IDI Bright Sparks Scholarship; AP is supported by an NHMRC Career Development Award; and JES is supported by an NHMRC Senior Research Fellowship.

Duality of interest The authors declare that there is no duality of interest associated with this manuscript.

Contribution statement LH performed data analysis and interpretation, and wrote the first draft of the manuscript; JLH assisted in data preparation and analysis, and reviewed and revised the manuscript; AP made a substantial contribution to data analysis and interpretation, and reviewed and revised the manuscript; and JES and DJM conceived the study, made substantial contributions to data interpretation, and reviewed and revised the manuscript. All authors approved the final version of the article. JES and DJM are the guarantors of this work and, as such, had full access to all of the study data and take responsibility for data integrity and the accuracy of data analysis. 


\section{References}

1. Ioacara S, Lichiardopol R, Ionescu-Tirgoviste $\mathrm{C}$ et al (2009) Improvements in life expectancy in type 1 diabetes patients in the last six decades. Diabetes Res Clin Pract 86:146-151

2. Morimoto A, Onda Y, Nishimura R et al (2013) Cause-specific mortality trends in a nationwide population-based cohort of childhood-onset type 1 diabetes in Japan during 35 years of follow-up: the DERI Mortality Study. Diabetologia 56:2171-2175

3. Allemann S, Saner C, Zwahlen M, Christ ER, Diem P, Stettler C (2009) Long-term cardiovascular and non-cardiovascular mortality in women and men with type 1 and type 2 diabetes mellitus: a $30-$ year follow-up in Switzerland. Swiss Med Wkly 139:576-583

4. Harding JL, Shaw JE, Peeters A, Guiver T, Davidson S, Magliano DJ (2015) Mortality trends among people with type 1 and type 2 diabetes in Australia: 1997-2010. Diabetes Care 38:733-734

5. Brown LJ, Scott RS, Moir CL (2001) All-cause mortality in the Canterbury (New Zealand) insulin-treated Diabetic Registry population. Diabetes Care 24:56-63

6. Lind M, Svensson AM, Kosiborod M et al (2014) Glycemic control and excess mortality in type 1 diabetes. N Engl J Med 371:1972-1982

7. Skrivarhaug T, Bangstad HJ, Stene LC, Sandvik L, Hanssen KF, Joner G (2006) Long-term mortality in a nationwide cohort of childhood-onset type 1 diabetic patients in Norway. Diabetologia 49:298-305

8. Goodkin G (1975) Mortality factors in diabetes. A 20 year mortality study. J Occup Med 17:716-721

9. Manuel DG, Schultz SE (2004) Health-related quality of life and health-adjusted life expectancy of people with diabetes in Ontario, Canada, 1996-1997. Diabetes Care 27:407-414

10. Narayan KM, Boyle JP, Thompson TJ, Sorensen SW, Williamson DF (2003) Lifetime risk for diabetes mellitus in the United States. JAMA 290:1884-1890

11. Miller RG, Secrest AM, Sharma RK, Songer TJ, Orchard TJ (2012) Improvements in the life expectancy of type 1 diabetes: the Pittsburgh Epidemiology of Diabetes Complications study cohort. Diabetes 61:2987-2992

12. Livingstone SJ, Levin D, Looker HC et al (2015) Estimated life expectancy in a Scottish cohort with type 1 diabetes, 2008-2010. JAMA 313:37-44

13. Australian Institute of Health and Welfare Diabetes (2009) Prevalence in Australia. An assessment of national data sources. Australian Institute of Health and Welfare, Canberra

14. Kenny SJAR, Geiss LS (1995) Prevalence and incidence of noninsulin-dependent diabetes, 2nd edn. U.S. Govt. Printing Office, Washington, DC
15. Loh V, Harding J, Koshkina V, Barr E, Shaw J, Magliano D (2014) The validity of self-reported cancer in an Australian population study. Aust N Z J Public Health 38:35-38

16. Harding JL, Shaw JE, Peeters A, Cartensen B, Magliano DJ (2015) Cancer risk among people with type 1 and type 2 diabetes: disentangling true associations, detection bias, and reverse causation. Diabetes Care 38:264-270

17. Chiang CL (1984) Chapter 7. The life table and its construction the abridged life table. The life table and its applications. Robert E Krieger Publishing Company, Malabar, pp 137-152

18. Andreev EM, Shkolnikov VM (2010) Spreadsheet for calculation of confidence limits for any life table or healthy-life table quantity. MPIDR Tech Rep 2010-005

19. Arriaga EE (1984) Measuring and explaining the change in life expectancies. Demography 21:83-96

20. Auger N, Feuillet P, Martel S, Lo E, Barry AD, Harper S (2014) Mortality inequality in populations with equal life expectancy: Arriaga's decomposition method in SAS, Stata, and Excel. Ann Epidemiol 24:575-580

21. Gregg EW, Cheng YJ, Saydah S et al (2012) Trends in death rates among U.S. adults with and without diabetes between 1997 and 2006: findings from the National Health Interview Survey. Diabetes Care 35:1252-1257

22. Gordon-Dseagu VL, Shelton N, Mindell JS (2013) Epidemiological evidence of a relationship between type-1 diabetes mellitus and cancer: a review of the existing literature. Int $\mathrm{J}$ Cancer 132:501-508

23. Australian Institute of Health and Welfare (2011) Diabetes prevalence in Australia: detailed estimates for 2007-08. Australian Institute of Health and Welfare, Canberra

24. Koro CE, Bowlin SJ, Bourgeois N, Fedder DO (2004) Glycemic control from 1988 to 2000 among U.S. adults diagnosed with type 2 diabetes: a preliminary report. Diabetes Care 27:17-20

25. Maclaine GD, Macarthur EB, Heathcote CR (1992) A comparison of death certificates and autopsies in the Australian Capital Territory. Med J Aust 156(462-463):466-468

26. Sington JD, Cottrell BJ (2002) Analysis of the sensitivity of death certificates in 440 hospital deaths: a comparison with necropsy findings. J Clin Pathol 55:499-502

27. Ravakhah K (2006) Death certificates are not reliable: revivification of the autopsy. South Med J 99:728-733

28. Harriss LR, Ajani AE, Hunt D et al (2011) Accuracy of national mortality codes in identifying adjudicated cardiovascular deaths. Aust N Z J Public Health 35:466-476

29. Beltran-Sanchez H, Preston SH, Canudas-Romo V (2008) An integrated approach to cause-of-death analysis: cause-deleted life tables and decompositions of life expectancy. Demogr Res 19:1323 